\title{
Sensory Change
}

National Cancer Institute

\section{Source}

National Cancer Institute. Sensory Change. NCI Thesaurus. Code C157429.

An alteration in the perceptual senses an individual. 